\title{
New observations in neuroscience using superresolution microscopy
}

\author{
Michihiro Igarashi, ${ }^{1 \star}{ }^{\circledR}$ Motohiro Nozumi, ${ }^{1}$ Ling-Gang Wu, ${ }^{2}$ Francesca Cella Zanacchi, ${ }^{3}$ ○István Katona, ${ }^{4}$ \\ (-)László Barna, ${ }^{5}$ Pingyong Xu, ${ }^{6,7,8}$ Mingshu Zhang, ${ }^{6,7}$ Fudong Xue, ${ }^{6,7}$ and Edward Boyden ${ }^{9}$ \\ ${ }^{1}$ Department of Neurochemistry and Molecular Cell Biology, Niigata University Graduate School of Medicine, Niigata 951-8510, Japan, ${ }^{2}$ National Institute \\ of Neurological Disorders and Stroke, Bethesda, Maryland 20892, ${ }^{3}$ Department of Nanophysics, Fondazione Istituto Italiano di Tecnologia, Genova 16163 , \\ Italy, ${ }^{4}$ Momentum Laboratory of Molecular Neurobiology, Department of Molecular and Developmental Neurobiology, Institute of Experimental Medicine, \\ Hungarian Academy of Sciences, Budapest 1083, Hungary, ${ }^{5}$ Nikon Center of Excellence for Neuronal Imaging, Institute of Experimental Medicine, \\ Hungarian Academy of Sciences, Budapest, Hungary, ${ }^{6}$ Key Laboratory of Noncoding RNA, Institute of Biophysics, Chinese Academy of Sciences, Beijing \\ 100101, China, ${ }^{7}$ Beijing Key Laboratory of Noncoding RNA, Institute of Biophysics, Chinese Academy of Sciences, Beijing, 100101, China, ${ }^{8}$ University of \\ Chinese Academy of Sciences, Beijing 100049, China, and ${ }^{9}$ Department of Biological Engineering and Brain and Cognitive Sciences, Media Laboratory, \\ Massachusetts Institute of Technology (MIT), Cambridge, Massachusetts 02139
}

Superresolution microscopy (SM) techniques are among the revolutionary methods for molecular and cellular observations in the $21^{\text {st }}$ century. SM techniques overcome optical limitations, and several new observations using SM lead us to expect these techniques to have a large impact on neuroscience in the near future. Several types of SM have been developed, including structured illumination microscopy (SIM), stimulated emission depletion microscopy (STED), and photoactivated localization microscopy (PALM)/stochastic optical reconstruction microscopy (STORM), each with special features. In this Minisymposium, experts in these different types of SM discuss the new structural and functional information about specific important molecules in neuroscience that has been gained with SM. Using these techniques, we have revealed novel mechanisms of endocytosis in nerve growth, fusion pore dynamics, and described quantitative new properties of excitatory and inhibitory synapses. Additional powerful techniques, including single molecule-guided Bayesian localization SM (SIMBA) and expansion microscopy $(\mathrm{ExM})$, alone or combined with super-resolution observation, are also introduced in this session.

\section{Introduction}

The molecular diversity inherent in brain is much larger than in other organs. For example, molecular and cellular neuroscience techniques have revealed a multitude of synapse-associated proteins that shape basic neuronal transmission. To understand the precise roles of these proteins, knowledge of the localization and positional relationships is essential. Unfortunately, the diffraction limit of conventional optical microscopy ( $200 \mathrm{~nm}$ ) precludes analyses of such information, because neuronal structural components are highly compressed within the narrow areas of synapses. In this Minisymposium, experts describe their experiences with innovative devices for super-resolution microscopy (SM) devised in the this century. We also introduce two additional associated techniques. These methods all use different principles to overcome optical limitations of conventional microscopy and are as described below.

Structured illumination microscopy (SIM). SIM can reconstruct the fine structure of cells by calculating the interference (moiré) pat-

Received July 31, 2018; revised Sept. 12, 2018; accepted Sept. 17, 2018.

Acknowledgments: M.I, L-G.W., F.C.Z., I.K., P.X., and E.B. thank our lab members for support. F.C.Z. acknowledge A. Barberis and the lab of Synaptic Plasticity of Inhibitory Networks at the Italian Institute of Technology for fruitful collaboration.

*To whom correspondence should be addressed: Michihiro Igarashi, MD, PhD, Department of Neurochemistry and Molecular Cell Biology, Niigata University, 1-757 Asahimachi, Chuo-ku, Niigata 951-8510, Japan. E-mail: tarokaja@med.niigata-u.ac.jp

https://doi.org/10.1523/JNEUROSCl.1678-18.2018

Copyright $\odot 2018$ the authors $\quad 0270-6474 / 18 / 389459-09 \$ 15.00 / 0$ terns induced by irradiation with striped-pattern excitation light (Gustafsson, 2000). With SIM, approximately $100 \mathrm{~nm}$ in lateral dimensions and $300 \mathrm{~nm}$ in the axial dimension can be visualized. Super-resolution images are easy to obtain with SIM, as it utilizes typical fluorescent dyes (Demmerle et al., 2017; Richter et al., 2018).

Stimulated emission depletion (STED) microscopy (Hell and Wichmann, 1994). A typical single-point scanning STED microscope superimposes the excitation beam with a doughnut-shaped STED beam to instantly quench molecules at the periphery of the excitation spot, thereby confining fluorescence emission to the doughnut center (Hell, 2007). This method of saturated quenching produces a fluorescent spot far below the diffraction limit to yield a subdiffraction resolution image. As a result, STED microscopy offers high temporal and spatial resolution (Hell, 2007), reaching approximately $60 \mathrm{~nm}$ every $26 \mathrm{~ms}$ as described in a study of fusion pore dynamics (Shin et al., 2018).

Stochastic optical reconstruction microscopy (STORM)/photoactivated localization microscopy (PALM). In PALM, individual fluorescent protein molecules are repeatedly photoactivated with a low-intensity light, imaged, and photobleached (Shcherbakova et al., 2014). The super-resolution image in PALM is reconstructed by merging all the localized single-molecule positions. PALM imaging combined with clustering analyses can be used to investigate in a more quantitative manner the scaffold protein distribution at the molecular level.

Single molecule-guided Bayesian localization (SIMBA) SM: Three remarkable advantages of SIMBA are its (i) exceptional spatial (50 

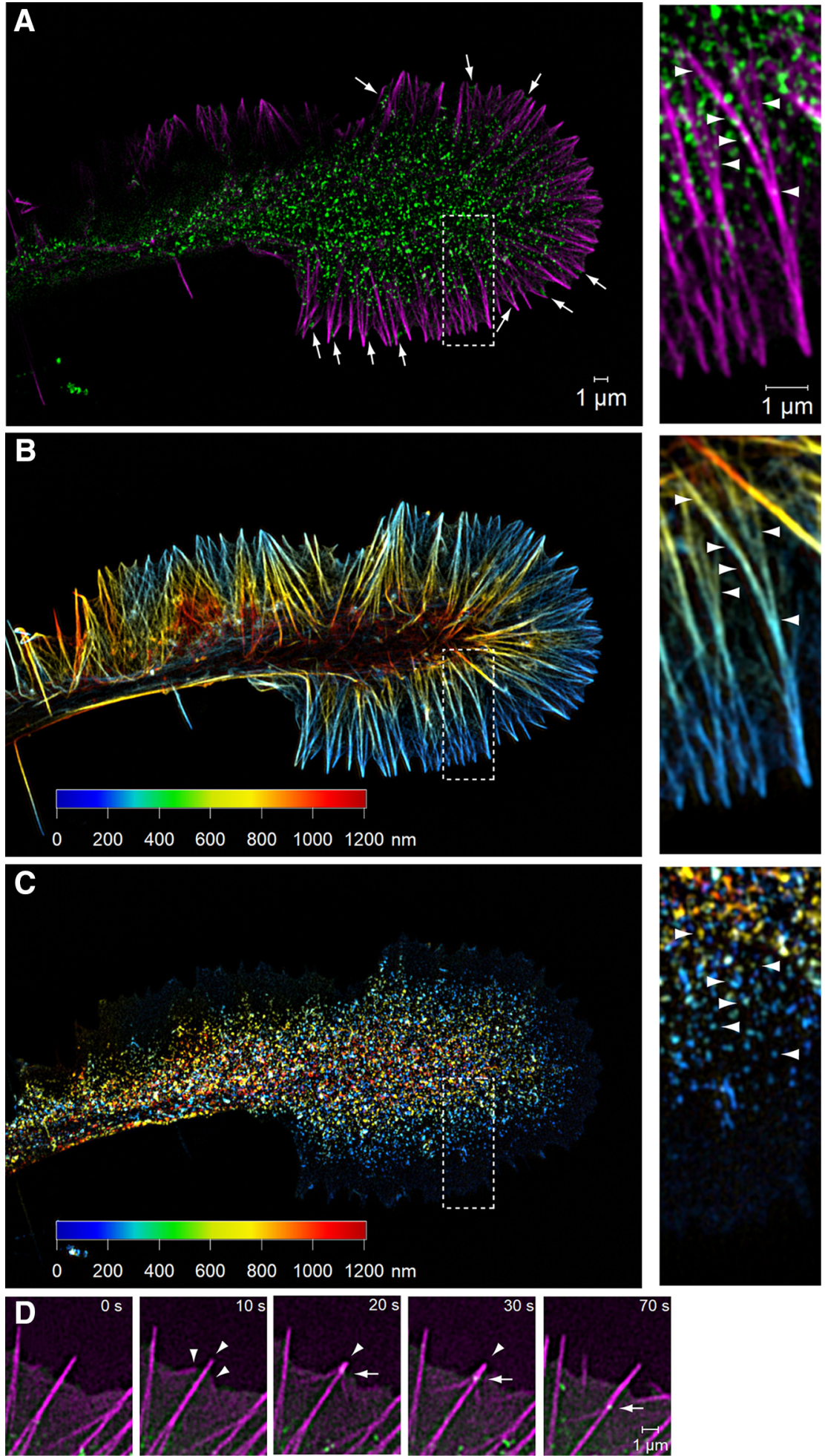

Figure 1. SIM reveals intracellular 3D distributions of individual actin filaments and vesicles labeled with GFP-synaptophysin in growth cones. (A) Left, F-actin (magenta) and GFP-synaptophysin (GFP-Syp; green) in a glutaraldehyde-fixed growth cone of a neuroblastoma NG108-15 cell. Right, enlarged image of the region marked by the dashed box on the left. The focus plane on the right was $330 \mathrm{~nm}$ higher than on the left, which focuses on the bottom of the growth cone. The GFP-Syp signals were detected in the vicinity of the filopodia (arrows in the left panel) and actin bundles (arrowheads in the right panel). (B) Color maps repsenting the height of F-actin from the substrate, in the growth cone shown in (A). Note that the central (C-) domain is obviously thicker than the peripheral (P-) domain. Most actin bundles are red in the C-domain, indicating that they are farther from the substrate. The color/height gradually decreases from the C-domain to the leading edge, suggesting that F-actin bundles are distributed along the dorsal/apical surface, but not along the basal surface, in the growth cone (Nozumi and lgarashi, 2017) (C) Color maps representing the height of GFP-Syp from the substrate in the growth cone shown in (A). By comparing the color map of GFP-Syp with that of F-actin, it is possible to determine which Syp-positive vesicles were close to F-actin bundles, which side of the bundle they were on, and what shape they have (arrowheads in the right panel). (D) SIM images of GFP-Syp accumulation coinciding with F-actin bundling (arrows). The 3D-SIM and live imaging showed Syp assembled in the vicinity of F-actin bundles at the leading edge (Nozumi et al., 2017). Arrowheads: three actin bundles forming into a thicker filopodium. 

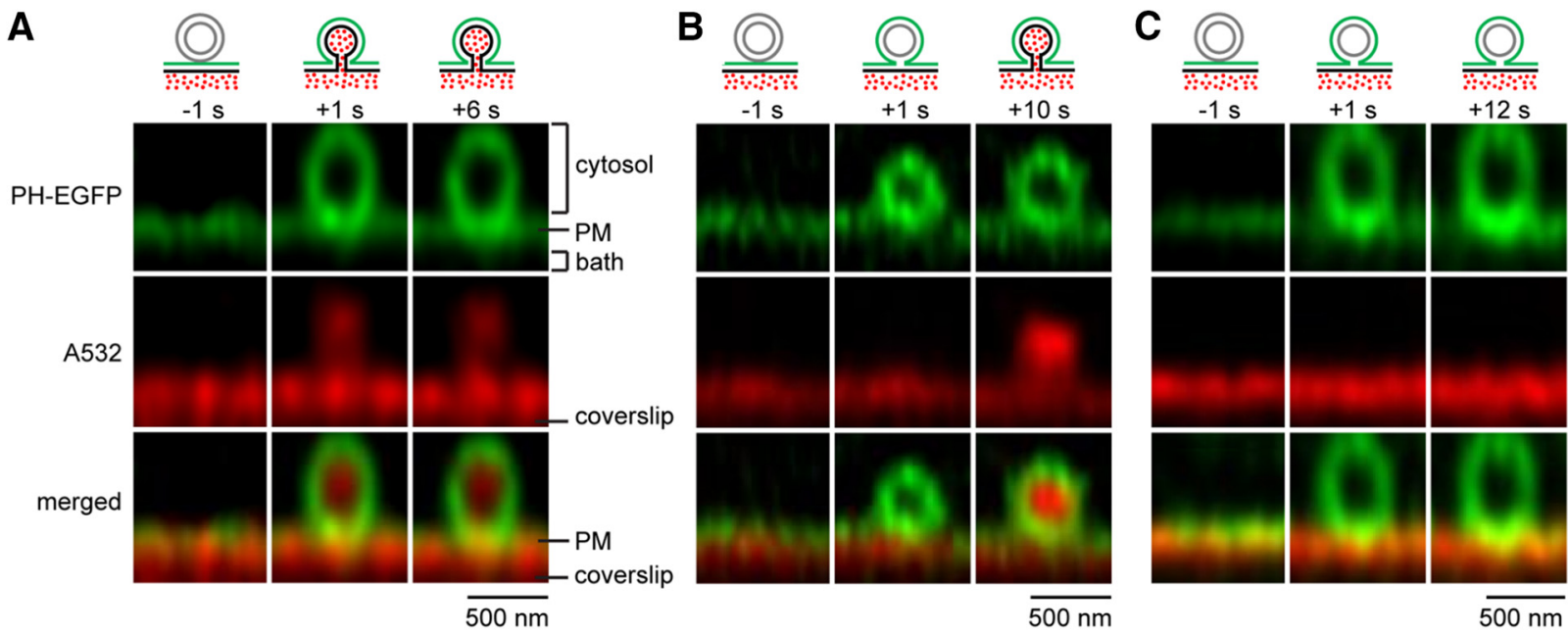

D

$X Z$ images: Pore
E Y: $250 \mathrm{~nm} \quad$ Line profile

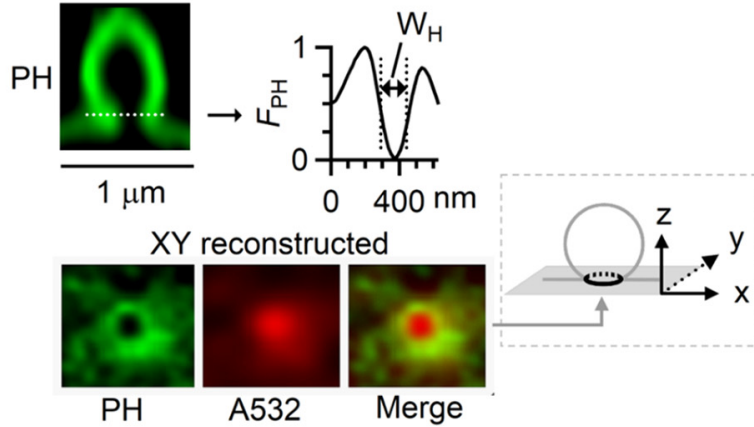

G

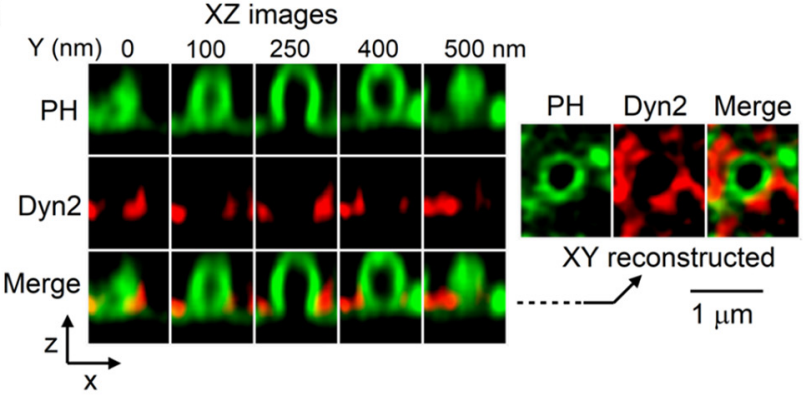

H

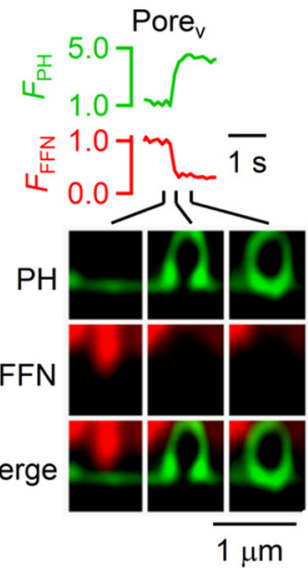

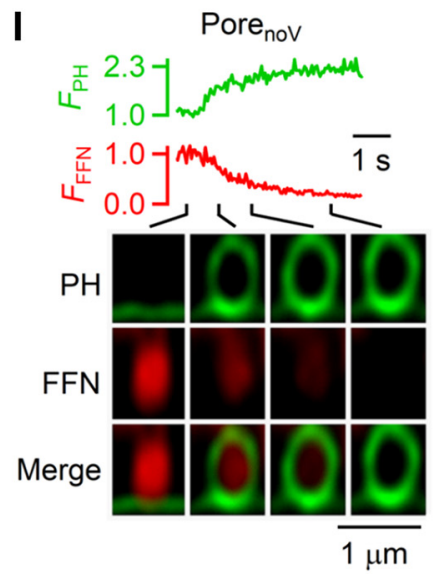

Figure 2. Visualizing the dynamics of hemi-fusion, fusion pore opening and vesicular-content release with STED microscopy. (A-C) STED XZ images PH (green, labeling inner leaflet) and A532 (red, in the bath) ( $\boldsymbol{A}, \mathrm{PH}$ and $\mathrm{A} 532$ appear in the W-profile at the same time), $\mathrm{PH}$-earlier ( $\boldsymbol{B}, \mathrm{PH}$ appears before A532, indicating hemi-fusion, and $\mathrm{A} 532$ appears later, indicating fusion pore has opened. (C) $\Omega$-profile labeled with PH only, indicating hemi-fusion. Labeled times are relative to depol ${ }_{15}$ onset. Cytosol, PM, bath, and coverslip location are labeled in A. (Figure legend continues.) 

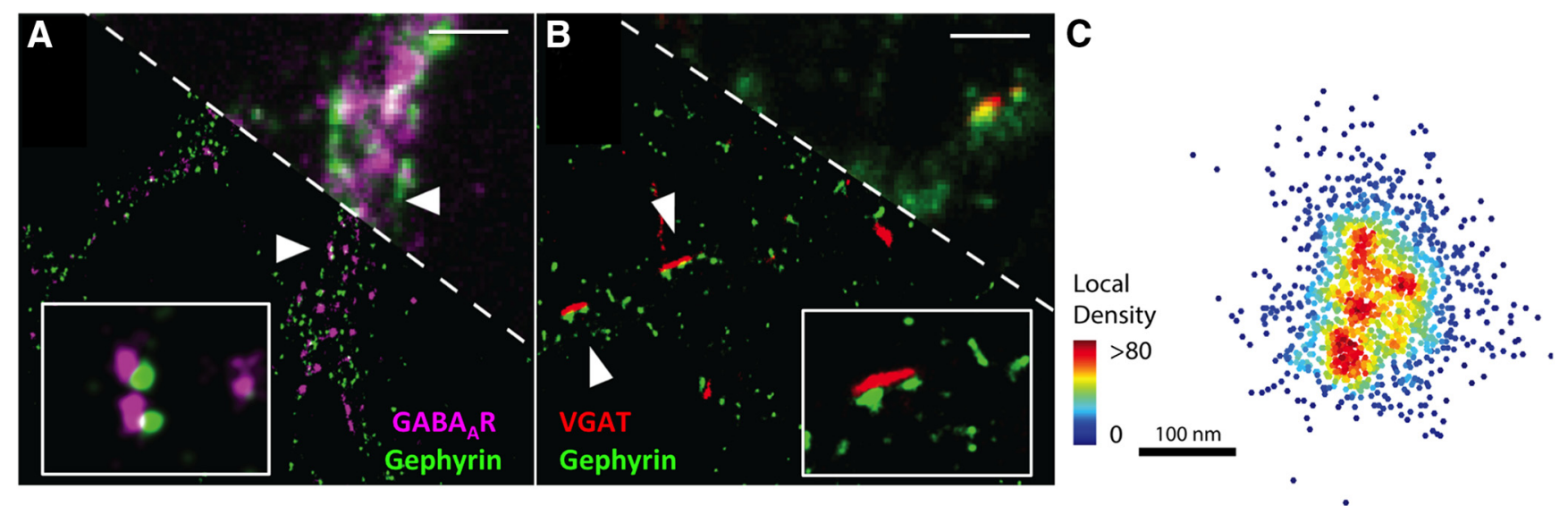

Figure 3. STORM Superresolution imaging of gephyrin scaffold proteins. Dual color STORM imaging of gephyrin associated with GABA $A_{A}$ receptors (A, inset) and the pre-synaptic marker VGAT (B, inset). Conventional and STORM images of Gephyrin stained with Alexa647 along with either $\mathrm{GABA}_{\mathrm{A}} \mathrm{R} \alpha 1$-subunit (left panel) or v-GAT (right panel) stained with Atto 520. Scale bar $2 \mu \mathrm{m}$ (A) (B). Axial dimension of the box $2 \mu \mathrm{m}$ (inset). Representation of gephyrin local density (radius $\sim 15 \mathrm{~nm}$ ) reveals the nanoscale organization of synaptic gephyrin in mono-spot and multi-spot clusters (C). Scale bars, $100 \mathrm{~nm}$. Image adapted from Pennacchietti et al. (2017). Sample credit: A. Barberis (Italian Institute of Technology).

$\mathrm{nm})$ and temporal (0.5 s/image) resolution, (ii) large field of view, and (iii) applicability for long-term live-cell imaging.

Expansion microscopy (ExM): Whereas current SM methods are difficult to apply to large or thick three-dimensional specimens, ExM uses the isotropic swelling of a dense, crosslinked polymer network of polyelectrolyte hydrogel materials to which key biomolecules or labels (e.g., GFP, antibodies) are covalently anchored. The addition of water to the preserved specimen causes the hydrogel to swell enormously $(\sim 4.5 \times$ in linear extent or $\sim 100 \times$ in volume) (Chen et al., 2015; Chang et al., 2017; Karagiannis and Boyden, 2018).

In the sections below, we describe some novel insights gained by using these revolutionary methods as well as recent advances that strengthen SM techniques.

\section{SIM reveals associations between F-actin organization and membrane trafficking in growth cones}

Growth cones are highly motile structures at the tips of growing axons during neuronal development and regeneration. They guide axon growth via chemotaxis and they form synapses after reaching a target (Lowery and Van Vactor, 2009). The thick area of the central domain of growth cones is rich in microtubule and organelle, whereas the peripheral domain, comprising F-actin, is thinner (Fig. 1). In the peripheral domain, branched F-actin constructs a sheetlike protrusion, called a lamellipodium, while unbranched filaments bundle together to form a slender protrusion, called a filopodium, at the growth cone's leading edge. To better understand the molecular basis of neuronal growth, the precise relationship between F-actin and the plasma membrane must be defined. However, there has

$\begin{array}{ll}\text { (Figure legend continued.) } & \text { (D) STED XZ images of PH and A532 taken at 50-nm intervals along }\end{array}$ the $Y$-axis, showing a W-profile with a visible pore (Pore ${ }_{v}$ ). (E) Upper left, W-profile at $Y=250$ $\mathrm{nm}$ from panel $\boldsymbol{D}$; upper right, the fluorescence profile of the dotted line across the pore with $W_{H}$ (full-width-half-maximum) labelled. Lower, $X Y$ images reconstructed from panel $D$ at a Z -focal plane across the pore (dotted line in upper panel). Inset, drawing of reconstructed plane (gray). (F) PH-labelled W-profile fluorescence ( $\mathrm{F}_{\mathrm{PH}}$, normalized to baseline), A532 spot fluorescence $\left(\mathrm{F}_{532}\right)$, Porev WH, and sampled images at times indicated with lines showing a pore undergoing rapid opening and slow constriction. (G) Left, PH and dynamin 2-mTurquoise2 (Dyn2, pseudocolor) XZ-images at different Y-axis location showing dynamin surrounding the pore. Right, $X Y$-reconstructed images across the pore (dotted line). (H-I) Pore-dynamics control content release: $F_{P H}, F_{F F N}$ (FFN511 fluorescence), and PH/FFN511 images for $\Omega$-profile with Pore $(H)$ or without a visible pore ( Pore $_{\text {nov }}$, I). been little progress despite the 3D elucidation of the growth cone structure, because the peripheral domain is small and thin $(<1 \mu \mathrm{m})$.

Using SIM, we examined the precise localization of $\sim 100$ proteins identified by proteomic analysis of mammalian growth cone (Nozumi et al., 2009, 2017) (Fig. 1). Our analysis suggested that the bundling of $\mathrm{F}$-actin is linked with local endocytosis at the leading edge (Nozumi et al., 2017). The observation of growth cones by 3D-SIM showed novel 3D intracellular structures and associations that could not be imaged with sufficient resolution using conventional confocal microscopy.

\section{STED imaging reveals hemifusion and fusion pore dynamics}

in live cells

Fusion is thought to occur through two mechanisms: the merging of the proximal leaflets of two bilayers (hemi-fusion), followed by distal leaflets to open a pore, or alternatively, by the formation of a proteinlined pore followed by the merging of lipid bilayers (Wu et al., 2014). The intense debate between these two hypotheses has recently been resolved with STED microscopy at live cells(Chiang et al., 2014; Wen et al., 2016; Zhao et al., 2016). Bovine chromaffin cells were transfected with EGFP or mNeonGreen attached to phospholipase $\mathrm{C} \Delta$ $\mathrm{PH}$ domain $(\mathrm{PH})$ which labels the cytosolic-facing leaflet of the plasma membrane (PM). With Atto 532 (A532) is in the bath, fusion generates $\mathrm{PH}$-labeled $\Omega$-profiles containing A532 spots, due to $\mathrm{PH}$ diffusion in the membrane and A532 diffusion in the solution (Fig. 2A). PH-labeled $\Omega$-profiles can appear at the same time as the A532 spot or appear alone without A532 spot (Fig. 2A-C) These results indicate that hemi-fusion (PM and vesicular cytosolic-facing leaflet fusion) is the first step to fusion(Zhao et al., 2016).

Fusion opens a pore to release transmitters and the rate of opening controls the rate of release. However, fusion pore has not been observed in live cells. The regulatory mechanisms are thus poorly understood. STED microscopy of PH/A532 in live chromaffin cells revealed dynamic fusion pore containing $180-720 \mathrm{~nm}$ vesicles revealed dynamic fusion pore behaviors, including opening, expansion, constriction, and closure of $0-490 \mathrm{~nm}$ pores within $26 \mathrm{~ms}$ to seconds (Fig. 2D-G)(Shin et al., 2018). These dynamic pore behaviors determine the rate of release of vesicular contents, such as the fluorescent false neurotransmitter 511 (FFN511) or neuropeptide Y-EGFP (Fig. 2 H-I) (Shin et al., 2018). Dynamic pore behaviors are generated by competition between pore expansion mediated by F-actin-dependent tension and constriction mediated by calcium/ 
A Perisomatic Interneuron
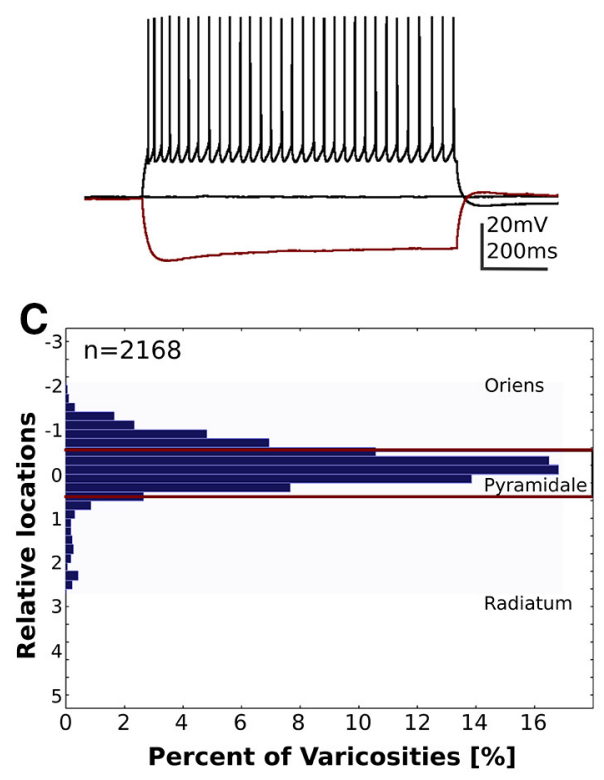
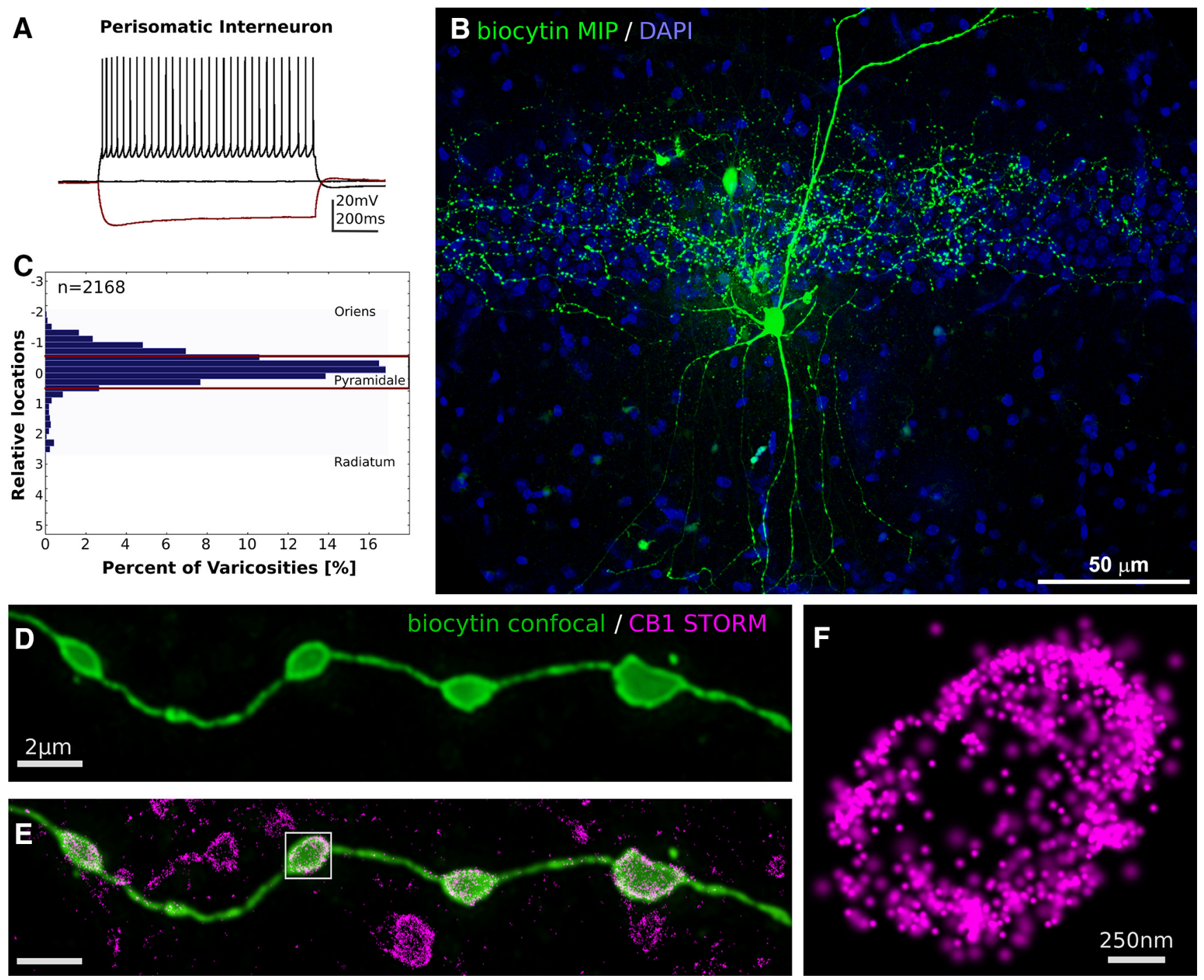

Figure 4. Combination of patch-clamp electrophysiology, confocal microscopy and STORM superresolution imaging. Visualization of individual target cells via biocytin injection during in vitro patch-clamp electrophysiological recording or during in vivo juxtacellular recording allows the correlated investigation of physiological, anatomical and nanoscale molecular parameters from the very same neuron within intact brain circuits. $(\boldsymbol{A})$ The representative voltage trace shows action potential firing of a hippocampal GABAergic interneuron recorded in the CA1 subfield of the mouse hippocampus. Note the regular spiking pattern, which is a typical physiological hallmark of $\mathrm{CB}_{1}$ cannabinoid receptor-positive interneurons. $(\boldsymbol{B})$ The neuron was filled with biocytin via the patch electrode during electrophysiological recording. Maximum intensity projection of the confocal image reveals the characteristic multipolar dendritic morphology of the interneuron, whereas the thin axons and the axon terminals are primarily restricted to the pyramidal layer. (C) The quantitative evaluation of the laminar bouton distribution confirms the identity of the cell as a perisomatically-targeting interneuron a.k.a. basket cell. Note that the vast majority of measured axonal varicosities $(n=2168)$ are located in the stratum pyramidale, whereas very few boutons are found in strata oriens and radiatum. The value " 0 " indicates the middle of the stratum pyramidale as reference point. (D) High-magnification confocal image of three axon terminals of the biocytin-filled basket cell. Terminals of the biocytin-filled basket cell. (E) STORM image of $C_{1} B_{1}$ receptorimmunostaining aligned with the confocal image of the biocytin-labeled axon demonstrates that most receptors are concentrated on the axon terminals of this $G A B A e r g i c$ interneuron. $(\boldsymbol{F})$ The vast majority of the STORM localization points representing $\mathrm{CB}_{1}$ receptors are accumulated along the contour of the bouton in accordance with the ubiquitous plasma membrane distribution of the receptor. [Modified from Dudok et al. 2015; figure kindly prepared by Dr. Barna Dudok].

dynamin(Shin et al., 2018). These findings provide the missing live-cell evidence proving the fusion-pore hypothesis, and establish a live-cell dynamic-pore theory accounting for fusion, fission and the regulation of these processes (Chiang et al., 2014; Shin et al., 2018).

SM for the study of GABAergic synaptic plasticity at the single-molecule level

Postsynaptic scaffolding proteins are key factors for the functional organization of synapses, and the mobility of the neurotransmitter receptors, with which these scaffolding molecules interact. At inhibitory synapses, gephyrin is present at a 1:1 ratio with receptors (Specht et al., 2013), suggesting that changes in gephyrin clustering may alter the number of receptors at the synapse and thus the strength of synaptic transmission. During inhibitory long-term potentiation induced by chemical stimulation, gephyrin redistributes from extrasynaptic to synaptic areas, thereby enhancing the clustering of synaptic $\mathrm{GABA}_{\mathrm{A}}$ receptors (Petrini et al., 2014). STORM is ideal for investigating single-molecule localization at the nanoscale level including the reorganization and distribution of synaptic receptors, such as $\mathrm{GABA}_{\mathrm{A}}$ receptors (Fig. 3A), and scaffold proteins (Pennacchietti et al., 2017), such as gephyrin (Fig. 3B), under conditions of plasticity. For more quantitative investigations of scaffold protein distribution, PALM imaging has recently been combined with clustering analyses showing the formation of gephyrin nanodomains at the synapse (Fig. 3C). 

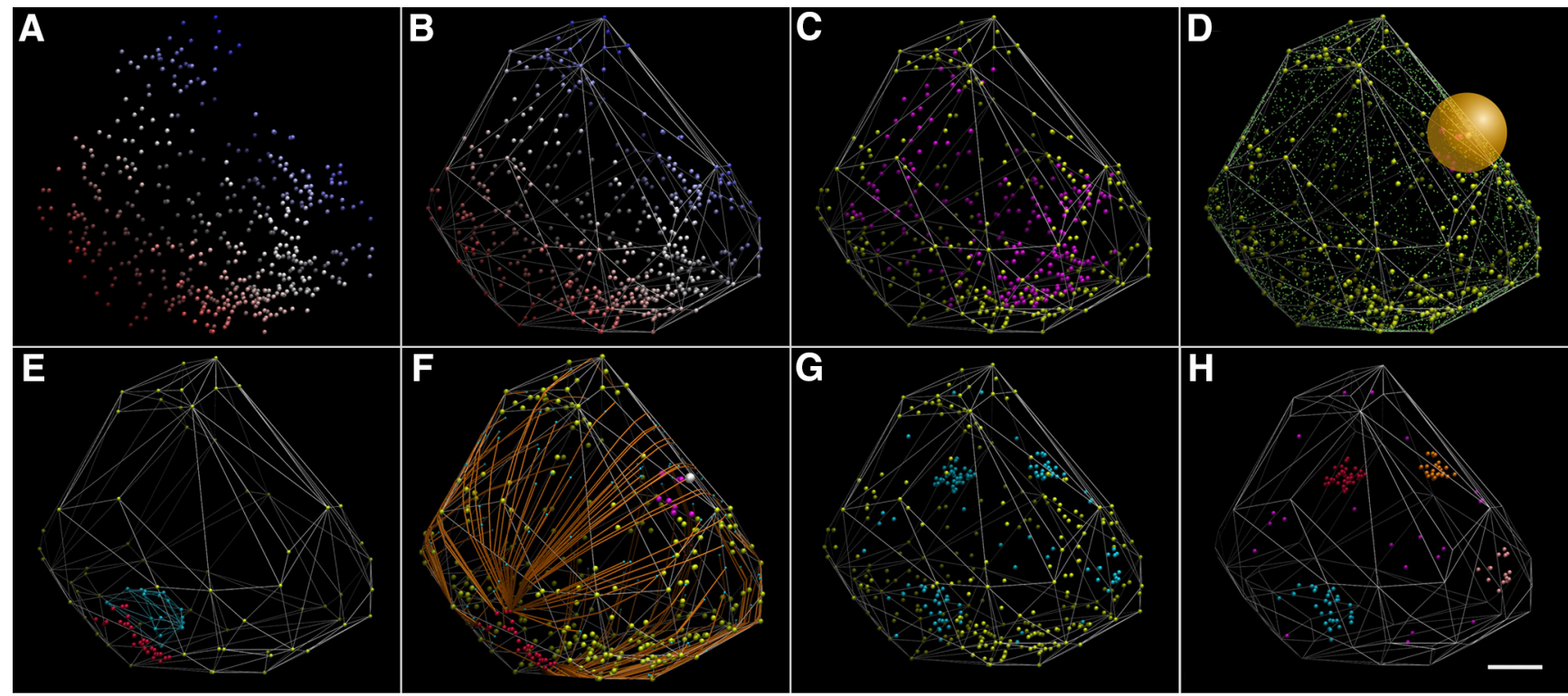

Figure 5. Handling nanoscale molecular information: Illustration by Visual Molecular Dynamics and data analysis by VividSTORM. (A) STORM super-resolution imaging generated localization points, which represent $\mathrm{CB}_{1}$ cannabinoid receptors visualized by immunofluorescence staining. These localizations all belong to a single $G A B A e r g i c$ axon terminal imaged in the $\mathrm{CA} 1$ subfield of the hippocampus. Note that other $\mathrm{CB}_{1}$ receptor-representing localizations belonging to neighboring cellular and subcellular profiles have been excluded from the image for clarity (see Fig. 4 for further details). The individual molecular localizations are illustrated as atoms with the help of the Visual Molecular Dynamics (VMD) software. The color depth encodes the z position of the receptor. All subsequent data analysis were performed by the VividSTORM software. (B) A convex hull was fitted onto the outermost STORM localizations (which become vertices) to approximate the plasma membrane of the axon terminals by exploiting the fact that $\mathrm{CB}_{1}$ receptors are densely and homogeneously cover the surface of GABAergic boutons. Chemical bonds in VMD are used to illustrate the convex hull edges. $(C)$ The distance of each receptor localizations can be measured from the convex hull faces. Yellow and magenta STORM localizations separate $C_{1}$ receptors along the surface or below the convex hull $(80 \mathrm{~nm})$. (D) Receptor density analysis is performed by selecting $C_{1}$ STORM localizations (yellow) and measuring randomly distributed reference points (little green dots) within a certain radius (large yellow sphere) of respective $\left(B_{1}\right.$ localizations. (E) Yellow STORM localizations depict the $\mathrm{CB}_{1}$ localizations constructing the convex hull. A single presynaptic active zone is visualized by STORM imaging of bassoon-immunostaining (cyan STORM localizations). Because bassoon is located $\sim 80 \mathrm{~nm}$ below the plasma membrane, the STORM localizations representing bassoon coordinates are projected onto the convex hull surface (red STORM localizations) to estimate the active zone position on the axon terminal membrane. $(\boldsymbol{F})$ Orange lines running along the surface of the convex hull link $\mathrm{CB}_{1}$ receptors ( yellow STORM localizations) with the closest localization on the active zone (red STORM localizations) providing an estimation of the nanoscale distance between the regulatory receptors and the location of their molecular effectors known to be present within the active zone. $(G) C_{1}$-containing GABAergic interneuron terminals form complex synapses and therefore carry multiple active zones. Visualization of all bassoon localizations (cyan STORM localizations) indeed suggest the presence of more than one bassoon cluster. $(\boldsymbol{H})$ In this particular bouton, 4 bassoon clusters (cyan, pink, orange and red localizations) were identified by DBSCAN, whereas other bassoon localizations identified as noise are depicted as magenta dots. Scale bar is $200 \mathrm{~nm}$; STORM localizations are shown as $17 \mathrm{~nm}$ spheres.

a

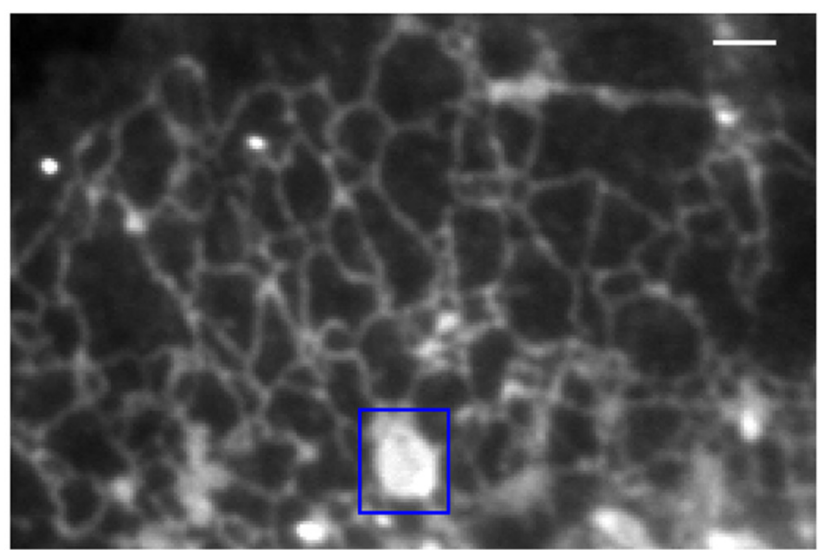

b

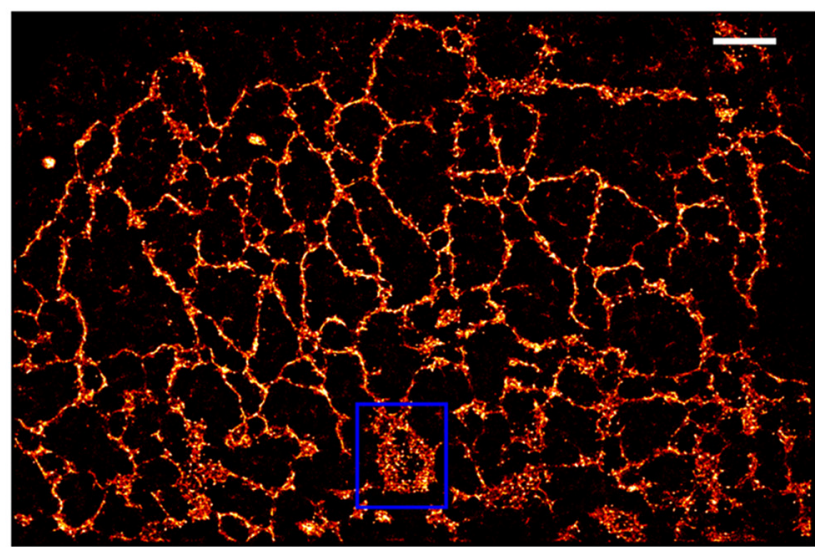

Figure 6. SIMBA analysis of endoplasmic reticulum structures in COS7 cells. $A, 0$ verlay of 200 total internal reflection fluorescence image frames representing diffraction-limit image of ER-Tracker Red-labeled endoplasmic reticulum. $\boldsymbol{B}$, Reconstructed SIMBA SR image of the same structure. Rectangle indicates the sheet structure. Scale bars: $2 \mu \mathrm{m}$.

\section{Nanoscale molecular imaging in brain circuits by STORM} superresolution imaging

STORM superresolution imaging has been used to demonstrate that the chemical synapse can be divided into functionally distinct nanodomains at both the presynaptic and postsynaptic sides (Dani et al., 2010; MacGillavry et al., 2013; Nair et al., 2013;
Specht et al., 2013; Tang et al., 2016; Sakamoto et al., 2018). We recently combined STORM superresolution imaging and confocal microscopy to measure molecular distributions at the nanoscale level in selected cells and in identified subcellular compartments (Fig. 4, 5; Dudok et al., 2015; Barna et al., 2016). A major advantage of combining confocal and STORM imaging is 


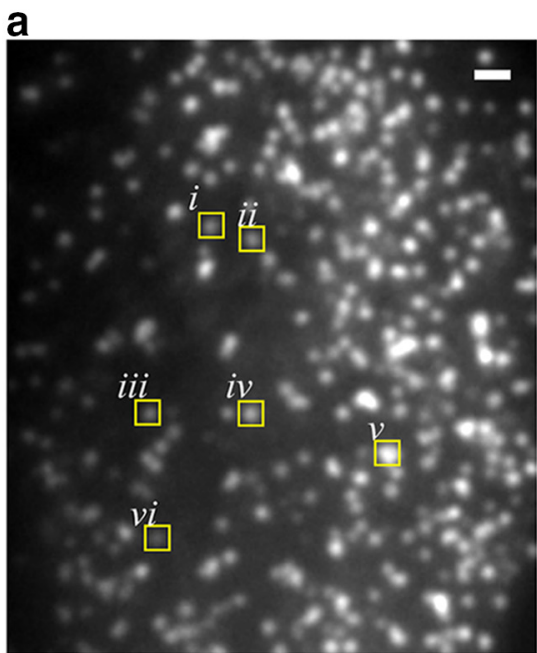

d

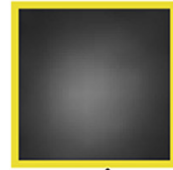

i

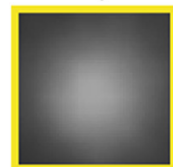

$i v$

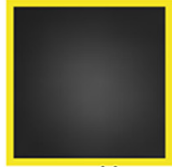

ii

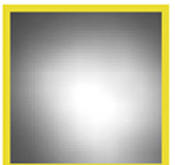

$v$

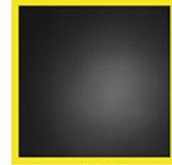

iii

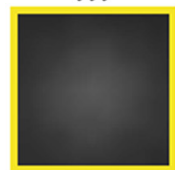

$v i$ b

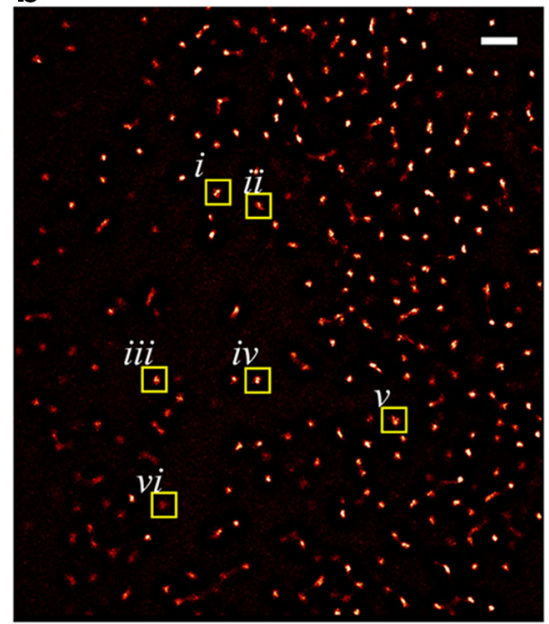

e

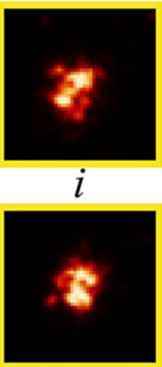

iv

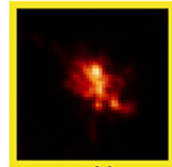

ii

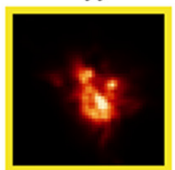

$v$

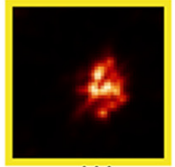

iii

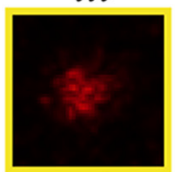

$v i$
C

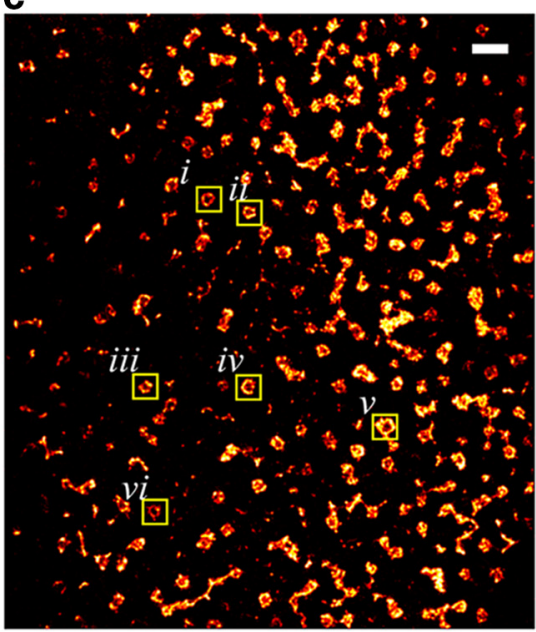

f

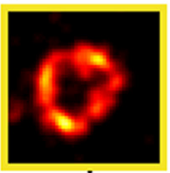

l

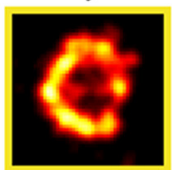

iv

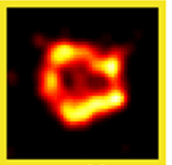

ii

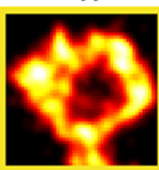

$v$

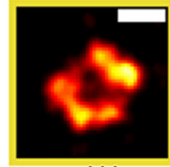

iii

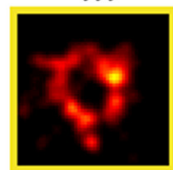

$v i$

Figure 7. SIMBA imaging of clathrin-coated pits in a live HeLa cell. (A) Overlay of 100 total internal reflection fluorescence image frames representing diffraction-limit image of $\mathrm{mE}$ Es 3.2 - clathrin light chain (CLC). (B) Reconstructed super-resolution radial fluctuations image of mEos3.2-CLC. (C) Reconstructed SIMBA image of mEos3.2-CLC. Scale bars: $1 \mu \mathrm{m}$. (D), (E), and (F): Zoomed-in images of clathrin-coated pits (CCPs) marked by yellow boxes in $(\boldsymbol{A}),(\boldsymbol{B})$, and, $(\boldsymbol{C})$ respectively. Scale bars: $200 \mathrm{~nm}$.

that it permits the study of molecular targets that are present abundant throughout tissues, but present in limited quantitites within the selected target cells and subcellular domains. This ability is due to the single-molecule nature of STORM imaging, which contributes to a substantially improved signal-to-noise ratio. This is an especially advantageous for distinguishing low intensity, but specific immunofluorescence labeling from the high autofluorescence background usually present in brain sections. By exploiting these advantages of correlated STORM and confocal microscopy, we recently to demonstrated the presence of ribosomes in the axon terminals of specific GABAergic interneuron types (Younts et al., 2016).

STORM imaging in combination with other microscopy methods and electrophysiological technologies will contribute to a better understanding of the cell-type-and subcellular compartmentspecific nanoscale molecular principles, which govern synaptic and other signaling mechanisms in brain circuits (German et al., 2017). In addition, STORM will help us understand the specific molecular changes underlying normal and abnormal plasticity processes in the brain.

\section{SIMBA SM}

Previously, we combined single-molecule localization and Bayesian analysis of blinking and bleaching to develop a new superresolution technique called SIMBA (Xu et al., 2017). This technique was used to examine the endoplasmic reticulum, an expansive and dynamic organelle comprising two types of structures, interconnected tubules and flattened sheets, according to models supported by conventional fluorescent microscopy. However, endoplasmic reticulum superresolution images reconstructed by SIMBA clearly show that the structures that appear as sheets by total internal reflection fluorescence microscopy are actually dense tubules surrounded by large empty areas (Fig. 6). The results are highly consistent with those of recent works by Lippincott-Schwartz and Blackstone (Nixon-Abell et al., 2016) in which a combination of five super-resolution technologies were used to demonstrate that "many structures previously proposed to be flat membrane sheets are instead densely packed tubular arrays." SIMBA imaging can clearly resolve mature clathrincoated pits as hollow rings (Fig. 7), whereas a recently developed technique utilizing superresolution radial fluctuations (SRRF) could not (Figure 7B, yellow boxes). As SIMBA requires fewer raw frames and is relatively insensitive to background fluorescence, it holds great potential for SR imaging of thick samples, such as brain slices, tissues and organisms in neuroscience with high spatial-temporal resolution.

\section{ExM}

Following the initial discovery that it was possible to isotropically swell a preserved biological specimen through hydrogel embedding, mechanical homogenization, and water swelling (Chen et al., 2015), three independent groups showed how to bind off-the-shelf chemicals to proteins, including labels such as GFP and fluorescent antibodies (Chozinski et al., 2016; Ku et al., 2016; Tillberg et al., 2016). These protein-retention forms of ExM have been applied to visualize a variety of proteins within synapses, axons, dendrites, and glia in brains of species ranging from Drosophila to mice and to humans. 

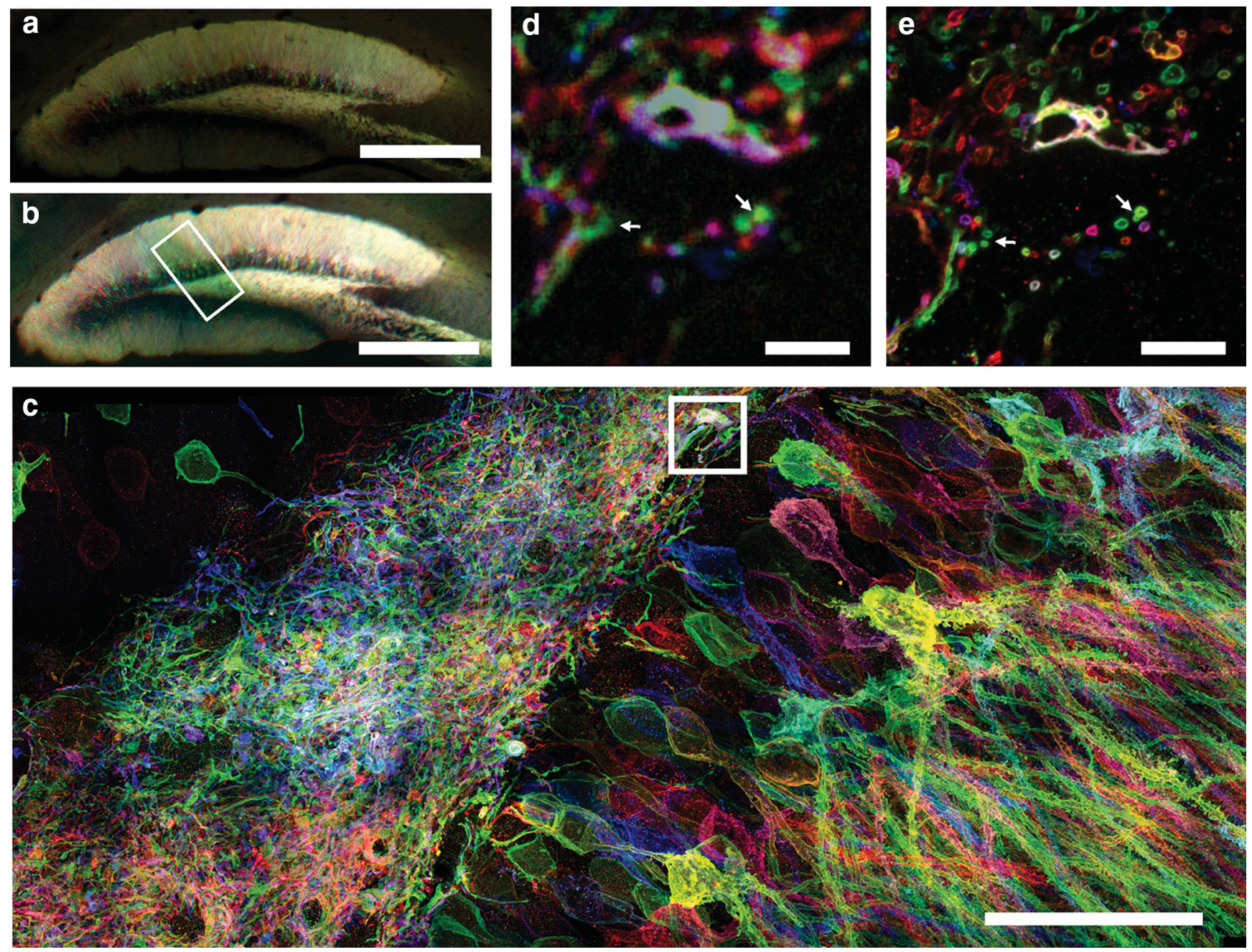

Figure 8. ExM of mouse brain circuitry. The expansion of the Brainbow-expressing mouse hippocampus. A, Low-magnification widefield fluorescence imaging showing immunostained mouse hippocampus expressing virally delivered Brainbow3.0. B, Postexpansion widefield image of the sample in panel $\boldsymbol{A}$. C, Maximum-intensity projection of high-resolution confocal microscopy stack following expansion of membrane-labeled Brainbow3.0 neurons from the boxed region in panel $\boldsymbol{B}$. $\boldsymbol{D}$, Pre-expansion confocal image showing one optical section of the boxed region in panel $\boldsymbol{C}$. $\boldsymbol{E}$ Postexpansion image of the same boxed region. ExM enables fine processes such as axons, which are not easily distinguished in dense neural circuitry (D), to be easily distinguished and traced (E). Scale bars: $\boldsymbol{A}, 500 \mu \mathrm{m} ; \boldsymbol{B}, 500 \mu \mathrm{m}$ (1,980 $\mu \mathrm{m}$ physical size); $\boldsymbol{C}, 5 \mu \mathrm{m} ; \boldsymbol{D}, 5 \mu \mathrm{m}$ (19.8 $\mu \mathrm{m}$ physical size), and $\boldsymbol{E}, 50 \mu \mathrm{m}$ (198 $\mu \mathrm{m}$ physical size). Adapted from Tillberg et al. (2016).

Given much interest in neural circuit tracing, or connectomics, ExM protocols have also been developed to enable mRNAs to be anchored to the swellable polymer, expanded away from each other, and then visualized with fluorescent in situ hybridization, a method in which individual molecules (i.e., mRNAs) are difficult to discern within thick intact tissues (Chen et al., 2015). Going forward, new kinds of polymer chemistry may enable even finer scale visualization, and new kinds of microscopes and algorithms may enable scalable imaging and analysis of increasingly large datasets from the brain, providing spatial precision across large-scale structures (Fig. 8).

\section{Discussion and Conclusions}

Since SM was first applied to neuroscience (Kittel et al., 2006; Willig et al., 2006; see also Tønnesen and Nägerl, 2013), more than ten years have passed. During this time, in addition to the results which we describe here, some of the important progress, such as the new structure of the axon initial segment (Xu et al., 2013) or the new methodology of the observation of living extracellular space in brain (Tønnesen et al., 2018), have been reported. SM enables precise visualization of endocytosis and exocytosis and quantitation of synaptic receptors, with further advances possible with new methods such as SIMBA and ExM.
SM should not be used for the simple confirmation of views derived from electron microscopy. Rather, SM can be used to unmask and resolve molecular structures and dynamics in physiological and pathological events. From this Minisymposium, we hope more neuroscientists will utilize these technologies, first to identify such events that cannot be observed in conventional microscopic fields and then to establish revolutionary concepts.

\section{References}

Barna L, Dudok B, Miczán V, Horváth A, László ZI, Katona I (2016) Correlated confocal and super-resolution imaging by VividSTORM. Nat Protoc 11:163-183. CrossRef Medline

Chang J-B, Chen F, Yoon Y-G, Jung EE, Babcock H, Kang J, Asano S, Suk H-J, Pak N, Tillberg PW, Wassie AT, Cai D, Boyden ES (2017) Iterative expansion microscopy. Nat Methods 14:593-599. CrossRef Medline

Chen F, Tillberg PW, Boyden ES (2015) Expansion microscopy. Science 347:543-548. CrossRef

Chiang HC, Shin W, Zhao WD, Hamid E, Sheng J, Baydyuk M, Wen PJ, Jin A, Momboisse F, Wu LG (2014) Post-fusion structural changes and their roles in exocytosis and endocytosis of dense-core vesicles. Nat Commun 5:3356. CrossRef

Chozinski TJ, Halpern AR, Okawa H, Kim H-J, Tremel GJ, Wong ROL, Vaughan JC (2016) Expansion microscopy with conventional antibodies and fluorescent proteins. Nat Methods 13:485-485. CrossRef 
Dani A, Huang B, Bergan J, Dulac C, Zhuang X (2010) Superresolution imaging of chemical synapses in the brain. Neuron 68:843-856. CrossRef Medline

Demmerle J, Innocent C, North AJ, Ball G, Müller M, Miron E, Matsuda A, Dobbie IM, Markaki Y, Schermelleh L (2017) Strategic and practical guidelines for successful structured illumination microscopy. Nat Protoc 12:988-1010. Medline

Dudok B, Barna L, Ledri M, Szabó SI, Szabadits E, Pintér B, Woodhams SG, Henstridge CM, Balla GY, Nyilas R, Varga C, Lee SH, Matolcsi M, Cervenak J, Kacskovics I, Watanabe M, Sagheddu C, Melis M, Pistis M, Soltesz I, Katona I (2015) Cell-specific STORM super-resolution imaging reveals nanoscale organization of cannabinoid signaling. Nat Neurosci 18:75-86. CrossRef Medline

German CL, Gudheti MV, Fleckenstein AE, Jorgensen EM (2017) Brain slice staining and preparation for three-dimensional super-resolution microscopy. Methods Mol Biol 1663:153-162. CrossRef Medline

Gustafsson MGL (2000) Surpassing the lateral resolution limit by a factor of two using structured illumination microscopy. J Microsc 198:82-87. CrossRef Medline

Hell SW, Wichmann J (1994) Breaking the diffraction resolution limit by stimulated emission: stimulated-emission-depletion fluorescence microscopy. Opt Lett 19:780-782. Medline

Hell SW (2007) Far-field optical nanoscopy. Science 316:1153-1158. CrossRef Medline

Karagiannis E, Boyden E (2018) Expansion microscopy: development and neuroscience applications. Curr Opin Neurobiol 50:56-63. CrossRef Medline

Kittel RJ, Wichmann C, Rasse TM, Fouquet W, Schmidt M, Schmid A, Wagh DA, Pawlu C, Kellner RR, Willig KI, Hell SW, Buchner E, Heckmann M, Sigrist SJ (2006) Bruchpilot promotes active zone assembly, $\mathrm{Ca}^{2+}$ channel clustering, and vesicle release. Science 312:1051-1054. CrossRef Medline

Ku T, Swaney J, Park J-Y, Albanese A, Murray E, Cho JH, Park Y-G, Mangena V, Chen J, Chung K (2016) Multiplexed and scalable super-resolution imaging of three-dimensional protein localization in size-adjustable tissues. Nat Biotechnol 34:973-981. CrossRef

Lowery LA, Van Vactor D (2009) The trip of the tip: understanding the growth cone machinery. Nat Rev Mol Cell Biol 10:332-343. CrossRef Medline

MacGillavry HD, Song Y, Raghavachari S, Blanpied TA (2013) Nanoscale scaffolding domains within the postsynaptic density concentrate synaptic AMPA receptors. Neuron 78:615-622. CrossRef Medline

Nair D, Hosy E, Petersen JD, Constals A, Giannone G, Choquet D, Sibarita JB (2013) Super-resolution imaging reveals that AMPA receptors inside synapses are dynamically organized in nanodomains regulated by PSD 95 . J Neurosci 33:13204-13224. CrossRef Medline

Nixon-Abell J, Obara CJ, Weigel AV, Li D, Legant WR, Xu CS, Pasolli HA, Harvey K, Hess HF, Betzig E, Blackstone C, Lippincott-Schwartz J (2016) Increased spatiotemporal resolution reveals highly dynamic dense tubular matrices in the peripheral ER. Science 354:aaf3928. CrossRef Medline

Nozumi M, Togano T, Takahashi-Niki K, Lu J, Honda A, Taoka M, Shinkawa $\mathrm{T}$, Koga H, Takeuchi K, Isobe T, Igarashi M (2009) Identification of functional marker proteins in the mammalian growth cone. Proc Natl Acad Sci U S A 106:17211-17216. CrossRef Medline

Nozumi M, Igarashi M (2017) Vesicular movements in the growth cone. Neurochem Int:1-6. CrossRef Medline

Nozumi M, Nakatsu F, Katoh K, Igarashi M (2017) Coordinated movement of vesicles and actin bundles during nerve growth revealed by superresolution microscopy. Cell Rep 18:2203-2216. CrossRef Medline

Pennacchietti F, Vascon S, Nieus T, Rosillo C, Das S, Tyagarajan SK, Diaspro A, Del Bue A, Petrini EM, Barberis A, Cella Zanacchi F (2017) Nanoscale molecular reorganization of the inhibitory postsynaptic density is a determinant of GABAergic synaptic potentiation. J Neurosci 37:1747-1756. CrossRef Medline
Petrini EM, Ravasenga T, Hausrat TJ, Iurilli G, Olcese U, Racine V, Sibarita JB, Jacob TC, Moss SJ, Benfenati F, Medini P, Kneussel M, Barberis A (2014) Synaptic recruitment of gephyrin regulates surface GABAA receptor dynamics for the expression of inhibitory LTP. Nat Commun 5:3921. CrossRef

Richter KN, Revelo NH, Seitz KJ, Helm MS, Sarkar D, Saleeb RS, D’Este E, Eberle J, Wagner E, Vogl C, Lazaro DF, Richter F, Coy-Vergara J, Coceano G, Boyden ES, Duncan RR, Hell SW, Lauterbach MA, Lehnart SE, Moser T, Outeiro TF, Rehling P, Schwappach B, Testa I, Zapiec B, Rizzoli SO (2018) Glyoxal as an alternative fixative to formaldehyde in immunostaining and super-resolution microscopy. EMBO J 37: 139-159. CrossRef Medline

Sakamoto H, Ariyoshi T, Kimpara N, Sugao K, Taiko I, Takikawa K, Asanuma D, Namiki S, Hirose K (2018) Synaptic weight set by Munc13-1 supramolecular assemblies. Nat Neurosci 21:41-49. CrossRef Medline

Shcherbakova DM, Sengupta P, Lippincott-Schwartz J, Verkhusha VV (2014) Photocontrollable fluorescent proteins for superresolution imaging. Annu Rev Biophys 43:303-329. CrossRef Medline

Shin W, Ge L, Arpino G, Villarreal SA, Hamid E, Liu H, Zhao WD, Wen PJ, Chiang HC, Wu LG (2018) Visualization of membrane pore in live cells reveals a dynamic-pore theory governing fusion and endocytosis. Cell 173:934-945.e12. CrossRef Medline

Specht CG, Izeddin I, Rodriguez PC, El Beheiry M, Rostaing P, Darzacq X, Dahan M, Triller (2013) A quantitative nanoscopy of inhibitory synapses: counting gephyrin molecules and receptor binding sites. Neuron 79:308-321. Medline

Tang AH, Chen H, Li TP, Metzbower SR, MacGillavry HD, Blanpied TA (2016) A trans-synaptic nanocolumn aligns neurotransmitter release to receptors. Nature 536: 210-214. CrossRef Medline

Tillberg PW, Chen F, Piatkevich KD, Zhao Y, Yu C-C (Jay); English BP, Gao L, Martorell A, Suk H-J, Yoshida F, Ellen M, DeGennaro DHR, Gong G, Seneviratne U, Tannenbaum SR, Desimone R, Cai D, Boyden ES (2016) Protein-retention expansion microscopy of cells and tissues labeled using standard fluorescent proteins and antibodies. Nature Biotechnology 34 987-992. CrossRef Medline

Tønnesen J, Nägerl UV (2013) Superresolution imaging for neuroscience. Exp Neurol 242:33-40.

Tønnesen J, Inavalli VVGK, Nägerl UV (2018) Super-Resolution Imaging of the Extracellular Space in Living Brain Tissue. Cell 172:1108-1121.e15. CrossRef Medline

Wen PJ, Grenklo S, Arpino G, Tan X, Liao HS, Heureaux J, Peng SY, Chiang HC, Hamid E, Zhao WD, Shin W, Nareoja T, Evergren E, Jin Y, Karlsson R, Ebert SN, Jin A, Liu AP, Shupliakov O, Wu LG (2016) Actin dynamics provides membrane tension to merge fusing vesicles into the plasma membrane. Nat Commun 7:12604. Medline

Willig KI, Rizzoli SO, Westphal V, Jahn R, Hell SW (2006) STED microscopy reveals that synaptotagmin remains clustered after synaptic vesicle exocytosis. Nature 440:935-939. Medline

Wu LG, Hamid E, Shin W, Chiang HC (2014) Exocytosis and endocytosis: modes, functions, and coupling mechanisms. Annu Rev Physiol 76:301331. Medline

Xu K, Zhong G, Zhuang X (2013) Actin, spectrin, and associated proteins form a periodic cytoskeletal structure in axons. Science 339:452-456. Medline

Xu F, Zhang M, He W, Han R, Xue F, Liu Z, Zhang F, Lippincott-Schwartz J, Xu P (2017) Live cell single molecule-guided bayesian localization super resolution microscopy. Cell Res 27:713-716.

Younts TJ, Monday HR, Dudok B, Klein ME, Jordan BA, Katona I, Castillo PE (2016) Presynaptic protein synthesis is required for long-term plasticity of GABA release. Neuron 92:479-492. CrossRef

Zhao WD, Hamid E, Shin W, Wen PJ, Krystofiak ES, Villarreal SA, Chiang HC, Kachar B, Wu LG (2016) Hemi-fused structure mediates and controls fusion and fission in live cells. Nature 534:548-552. CrossRef Medline 